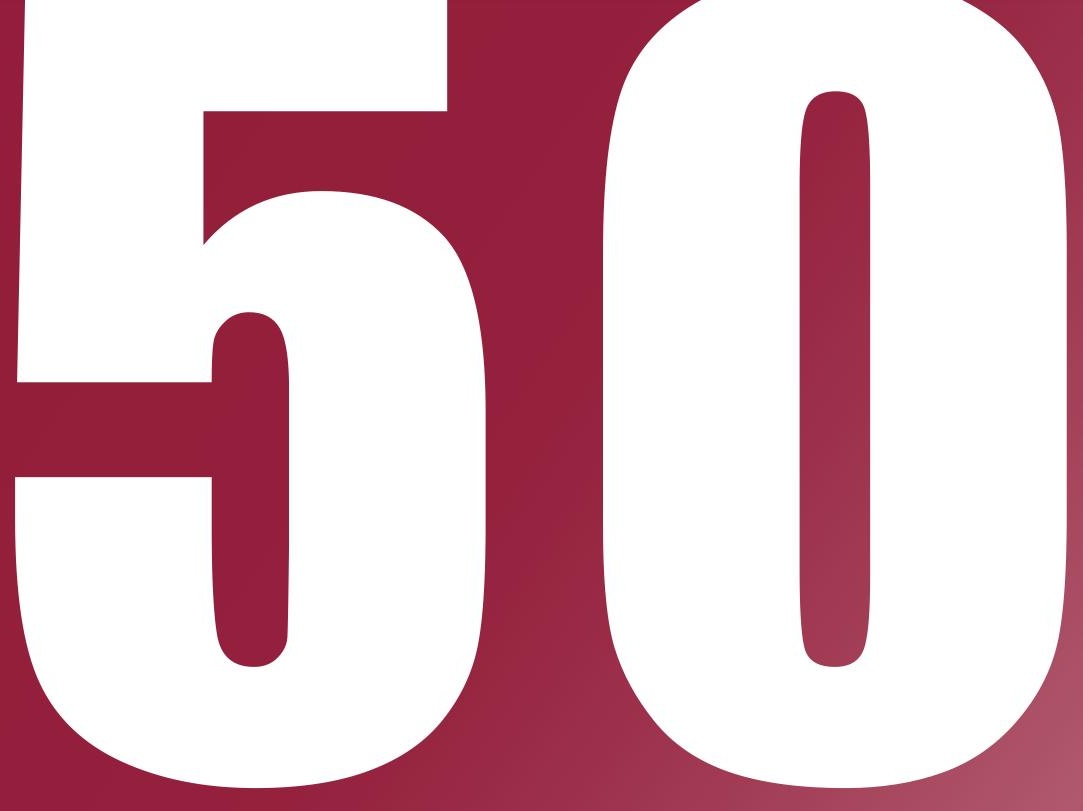

ÁMBITOS

REVISTA

INTERNACIONAL

DE

COMUNICACIÓN

$\mathbf{N}^{\circ} \mathbf{5 0}$

EDICIÓN OTOÑO

2020

ISSN: 1139-1979

E-ISSN: 1988-5733

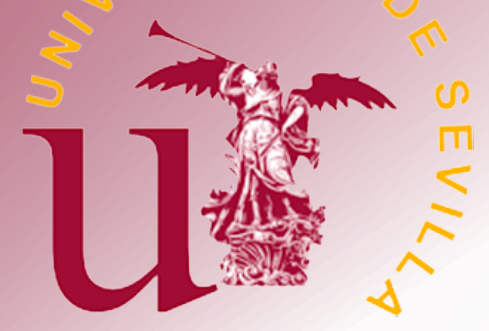




\section{INDICE}

EDITORIAL EDITORIAL

Presentación del Monográfico. Comunicación y juventud. Nuevos medios, representación, recepción y participación en contenidos de entretenimiento e información

Presentation of the monograph. Communication and youth. New media, representation, reception and participation in entertainment and information content.

Juan Francisco Gutiérrez Lozano, Francisco Javier Ruiz del Olmo

7-9

\section{MONOGRÁFICO MONOGRAPH}

El consumo audiovisual de la Generación Z. El predominio del vídeo online sobre la televisión tradicional The audiovisual consumption of Generation $Z$. The predominance of online video over traditional television

La imagen de los jóvenes en las estrategias transmedia de las series de televisión: el caso de Skam España en Instagram

The image of young people in transmedia strategies of TV series: the case of Skam España on Instagram

Sergio Jesús Villén Higueras, Francisco Javier Ruiz del Olmo

La penetración de Netflix en el público español ¿cuestiona el modelo televisivo tradicional?

Does the arrival of Netflix in the Spanish audience challenge the traditional television model?

Javier Bustos Díaz

49-61

Generación Z y consumo de información política: entre la televisión y los nuevos formatos mediáticos Generation $Z$ and consumption of political information: between television and new media formats

Ruth Gómez de Travesedo Rojas, Marta Gil Ramírez

$62-79$

Actualidad y comedia: El éxito de El Intermedio y La Resistencia entre los espectadores jóvenes

Information and comedy: The Success of El Intermedio and La Resistencia for young audiences

Inmaculada Concepción Aguilera García

80-95 
El efecto socializador de articular un espacio de comunidad virtual en el ecosistema del aula

The socializing effect of creating a virtual community space in the classroom environment

Lorea Ariadna Ruiz Gómez, Mónica Hinojosa Becerra, Francisco Javier Ruiz San Miguel

Juventude ciberativista e educação: reflexões sobre um jeito hacker de ser

Cyber activity youth and Education: reflections on a hacker way of being

Carla Azevedo de Aragão, Pietro Matheus Bompet Fontoura Alves, Karina Moreira Menezes

La risa grotesca en la obra de Bob Dylan: análisis de la Bacanal y el Festín Carnavalesco en la trilogía folk-rock eléctrica

Grotesque Laughter in Bob Dylan's Work: Analysis of The Bacchanal and the Carnival Feast in the Electric Folk-rock Trilogy

Jesús Albarrán Ligero

El papel del intertexto en el videojuego. Una partida, mil y una historias

Intertext in video games. A game, thousand and one stories

Rocío Serna-Rodrigo

145-158

\section{ÁmBitos PERSONALES PERSONAL ÁmBITOS}

El auge de Twitch: nuevas ofertas audiovisuales y cambios del consumo televisivo entre la audiencia juvenil The rise of Twitch: New audiovisual offers and the transformation of Television consumption among young audiences

\section{ARTÍCULOS ARTICLES}

La recepción de los medios de comunicación para residentes rusos en la Costa del Sol

Mass media reception by Russian residents on the Costa del Sol

Natalia Meléndez Malavé, José Carlos Pozo García

176-192

La calidad periodística en la cobertura de terremotos: Caso Ecuador

Journalistic quality in earthquake coverage: The case of Ecuador

Juan Pablo Arrobo-Agila, Mendoza María, Ignacio Aguaded

La credibilidad publicitaria en la nueva esfera mediática: los universitarios y los medios

Advertising credibility in the new media sphere: university students and the media 
Arco iris en medios brasileños: percepciones sociales sobre una campaña publicitaria LGBTQIA+

Rainbow in Brazilian media: social perceptions of an LGBTQIA+ advertising campaign

Leonardo Ferreira Batista, José Geraldo de Araújo Ramalho Filho

224-239

Rituales y comunicación política: la toma de posesión de los presidentes autonómicos españoles

Rituals and political communication: the Spanish autonomous Presidents inauguration

Ricardo Domínguez García

240-258

RESEÑAS REVIEWS

Una mirada diferente para hacer periodismo

A different look for making journalism

Guillermo Paredes-Otero

259-261

Periodismo y tecnología: una simbiosis continua

Journalism and technology: a continuous symbiosis

Luisa Graciela Aramburú Moncada

262-265

Una propuesta metodológica para impartir la asignatura de redacción periodística

Journalism and technology: a continuous symbiosis

Noelia Ruiz-Alba

266-268 


\title{
Rituales y comunicación política: la toma de posesión de los presidentes autonómicos españoles
}

\author{
Rituals and political communication: the Spanish autonomous Presidents \\ inauguration
}

\author{
Ricardo Domínguez García \\ Universidad de Sevilla.| Avda. Américo Vespucio S/N, 41092, Sevilla |España \\ | https://orcid.org/0000-0001-7325-1861| ricdomgar@alum.us.es
}

Fechas | Recepción: 26/05/2020 | Aceptación: 05/08/2020 | Publicación final: 15/10/2020

\begin{abstract}
Resumen
Uno de los eventos institucionales en el que se puede apreciar con mayor claridad el uso de símbolos para reforzar el estatus de poder de un gobernante es la toma de posesión. Se trata de un acto de carácter simbólico y repetitivo en el que un político asume su cargo y en ocasiones expone, de manera más o menos breve, las líneas maestras de su futura gestión. Esta investigación deja a un lado el contenido de los discursos que se puedan ofrecer en estas celebraciones, para centrarse en el uso de rituales y símbolos, como parte del relato de la comunicación política. Para ello, se han analizado las 203 tomas de posesión de los 106 presidentes que han tenido las 17 comunidades autónomas, mediante la metodología de análisis de contenido y el trabajo con el programa informático IBM SPSS Statistic. Así, se ha podido determinar que este acto oficial tiene una gran riqueza ritual y diversidad, ya que se adapta el ceremonial y la simbología de la toma de posesión a las particularidades histórico-políticas de cada territorio.
\end{abstract}

\begin{abstract}
One of the institutional events in which the use of symbols, with the objective of reinforcing the power status of a ruler, can be seen more clearly is the inauguration. It is an act of a symbolic and repetitive nature in which a politician takes office and sometimes exposes the main lines of his future management. This research sets aside the content of the speeches that can be offered in these celebrations, to focus on the use of rituals and symbols, as part of the message of political communication. To this end, we have analyzed the 203 inaugurations of the 106 presidents that the 17 Spanish autonomous communities have had, using the content analysis methodology and working with the IBM SPSS Statistic computer program. Thus, it has been possible to determine that this oficial act has great ritual richness, since the ceremonial and the symbolism of the inauguration is adapted to the historicalpolitical particularities of each territory.
\end{abstract}

\footnotetext{
Forma de citar:

Domínguez García, R. (2020). Rituales y comunicación política: la toma de posesión de los presidentes autonómicos españoles. Ámbitos. Revista Internacional de Comunicación 50, pp. 240-258. doi: 10.12795/Ambitos.2020.i50.16
} 
Palabras clave: comunicación política, Keywords: political communication, protocol, protocolo, toma de posesión, ceremonia, inauguration, ceremony, autonomous comunidades autónomas.

regions.

\section{INTRODUCCIÓN}

A lo largo de la historia, los gobernantes se han servido de símbolos y ritos con los que demostrar su poder sobre sus gobernados y reforzar así su autoridad para ejercer el poder. Muestra de ello es que los reyes han lucido coronas, los obispos mitras y los militares uniformes acordes a su posición jerárquica. Como se verá en este estudio, la política moderna ha buscado, del mismo modo, rodearse de distintivos, ceremonias y rituales con los que comunicar su poder a la sociedad.

Uno de los eventos institucionales en el que actualmente se puede apreciar con mayor claridad el uso de símbolos con el objetivo de reforzar el estatus de poder de un gobernante es la toma de posesión. Se trata de un acto de carácter simbólico y repetitivo en el que un político asume su cargo y en ocasiones expone, de manera más o menos breve, las líneas maestras de su futura gestión. Los estudios sobre Comunicación Política tienden tradicionalmente a primar lo que se dice sobre cómo se dice. Sin embargo, esta investigación deja a un lado el contenido de los discursos que se puedan ofrecer en estas celebraciones, para centrarse en el uso de rituales y símbolos, como parte del relato de la Comunicación Política.

A lo largo de este trabajo de investigación, se podrá observar que las ceremonias de toma de posesión de los presidentes autonómicos españoles han ayudado a construir un universo simbólico con el que se refuerza la identidad de cada comunidad autónoma y se fortalecen sus instituciones.

\section{MARCO TEÓRICO}

Con la aparición progresiva de nuevas formas de comunicación, los eventos políticos han ido transformándose, hasta convertirse en actos programados directamente para atraer a las cámaras y en los que prima el impacto visual, por encima incluso del propio discurso político. En este sentido, Thompson (2003) afirma que los medios de comunicación han aportado una mayor visibilidad a los actos institucionales, permitiendo que la ciudadanía pase de participar mediante la copresencia a hacerlo a distancia gracias a la publicidad mediática. Una derivada de esto es que en la actualidad ya no están pensados -solo- para los invitados presentes, sino también para los espectadores que los siguen en directo a través de los medios de comunicación o de Internet. Este fenómeno denominado invitado mediático (Laforet, 2013) hace que el protocolo de las ceremonias oficiales vea alterado muchas de sus formas tradicionales, con el objetivo de adaptarse a las exigencias de la comunicación política y ejemplo de ello es el uso de tarimas que eleven las zonas de honor o presidencias para facilitar un mejor tiro de cámara. Otros aspectos en los que ha influido lo que se podría denominar la mediatización del ceremonial es la incorporación de discursos políticos en todo tipo de actos institucionales, pensados ahora con el claro objetivo de conseguir situar un mensaje político, o los horarios de los actos, que ya tienen en cuenta las horas de emisión de los informativos. Sin embargo, se debe señalar que este proceso no conlleva que se incumplan las reglas establecidas por el protocolo. Así, el uso de las banderas en los actos institucionales puede servir para ilustrar esta afirmación. Su ordenación, está determinada por la Ley, por lo que nunca se pone en cuestión, pero sí su ubicación. De esta manera, el hecho de que de las banderas de un territorio se sitúen, o no, tras 
el atril desde donde se realizan las intervenciones se decide teniendo en cuenta criterios claramente comunicativos.

Esta concepción del protocolo visto desde la perspectiva de la comunicación ha generado una corriente académica que en España tiene como referentes a autores como Marín Calahorro (2000), Otero (2011), Fuente (2005) o Sierra (2008). Si se analiza desde la visión global que aporta la Comunicación Política, el Protocolo es el encargado de gestionar el proceso comunicativo que se produce entorno a los actos públicos institucionales, estableciendo la secuencia de elementos ceremoniales que les dan forma y ordenando jerárquicamente los símbolos y las autoridades presentes en ellos.

\subsection{El encaje teórico del ritual político}

La investigación del ritual cuenta con una larga y prolífica trayectoria académica. Sin embargo, generalmente se ha centrado en las culturas primitivas, pero no en las sociedades modernas. En un primer momento, los encargados de su estudio son los historiadores de la antigüedad clásica, y a partir de la mitad del siglo XIX, su interpretación y análisis sistemático queda a cargo de antropólogos como Arnold van Gennep. En el mundo académico no hay, al principio, un interés especial por abordar la dimensión política del ritual debido a que, tradicionalmente, éste es relacionado con lo sagrado y, por tanto, con la religión (Durkheim, 1912). De este modo, en los estudios sobre la política moderna, se observa frecuentemente el uso de símbolos y el comportamiento ritual como algo decorativo o complementario a las actividades políticas realmente importantes. No obstante, en la segunda mitad del siglo XX, un sector académico comienza a abordar el debate sobre la superación del concepto tradicional de ritual y la necesidad de estudiar su dimensión política. Así, el concepto de ritual adquiere una autonomía frente al campo religioso y se entiende que "sirve para manifestar el estatus del individuo en cuanto persona social en el sistema estructural en el que se encuentra en el momento actual" (Leach, 1977, p. 33).

Más adelante, entre finales de los años ochenta y mediados de los noventa, se publican algunas de las obras sobre las que se basa el concepto de ritual político y que restituyen la importancia de los símbolos y las prácticas sacralizadas en la política de las democracias modernas. Esta corriente tiene como principales representantes a Marc Abèlés (1988), David Kertzer (1988) y Georges Balandier (1994). Estos autores defienden que el ritual tiene una dimensión social que conlleva una relación con lo sagrado, pero no con la religión, sino con la evocación de valores trascendentes como la nación, la historia o la familia. De esta forma, en las sociedades actuales, donde el poder se ha disociado de lo sagrado, las manifestaciones clásicas son sustituidas por el espectáculo político. Además, es importante la matización que aporta Abèlés (p. 398) al afirmar que, aunque pueden existir rituales políticos modernos, estos no son un simple instrumento más dentro de la estrategia de comunicación política, sino que son una forma histórica de legitimación (p. 398).

Sin embargo, conviene matizar que todo acto político más o menos solemne no debe ser considerado automáticamente como un ritual. Así, nos debemos situar en una visión intermedia entre una definición demasiado restrictiva, que lo limitaría a la esfera religiosa o sobrenatural, y una definición demasiado amplia, que entiende como ritual a cualquier actividad humana estandarizada. Como afirma Kertzer (1988), "The sacred ultimately refers not to a supernatural entity, but rather to people's emotionally charged interdependence, their societal arrangements" (p. 9). Es la carga emotiva y la presencia de simbolismo lo que diferencia a un ritual de otras acciones estandarizadas y repetitivas, como los hábitos o costumbres.

Ámbitos. Revista Internacional de Comunicación | ISSN: 1139-1979 | E-ISSN: 1988-5733, №. 50. (2020) 
A este respecto, cabe señalar que, con la separación entre la Iglesia y el Estado, queda en evidencia el vacío que dejan las religiones tradicionales, y es la política quien debe ocupar ese hueco, sacralizando símbolos y ritos (Balandier, 1994). Por tanto, la secularización de la sociedad no conlleva la desacralización, sino la sustitución de los rituales de las religiones tradicionales por otros propios del Estado nación. Sin embargo, una vez más hay que insistir en que no toda ceremonia institucional es un ritual, ya que mientras los ritos están relacionados con los grandes asuntos que dan sentido a la sociedad, "las ceremonias cumplen funciones similares, con respecto a situaciones menos trascendentes, por lo que sería una especie de rito en tono menor" (Gómez García, 2002, p. 2).

\subsection{Características del ritual político}

Como se ha podido comprobar, el ritual político es un concepto algo difuso y se analiza desde diferentes perspectivas, por lo que este apartado debe entenderse como una orientación para entender el concepto, más que como un listado exhaustivo de requisitos:

1) Dimensión simbólica: Los rituales se construyen a partir de símbolos, y es la potencia de tales símbolos de donde surge el poder del ritual político (Kertzer, 1988). Los símbolos utilizados en los rituales pueden ser extraordinarios u ordinarios, pero usados de un modo especial, que sirven para focalizar la atención de los participantes y para provocar en ellos un sentimiento de emoción. Muchos símbolos son una realidad palpable, lo que les da una mayor potencia. Es el caso de las banderas, que no son un simple trozo de tela, sino que encarnan un concepto tan poderoso como la Nación. En el ritual político, los símbolos aportan identidad, reúnen a la gente y refuerzan la autoridad de quien los manipula. Como la autoridad es una abstracción en sí misma, es a través del simbolismo como se reconoce quiénes son los poderosos y, por ello, el ritual no expresa simplemente el poder, sino que lo constituye.

2) Repetición y fragmentación: Abélès, citando a Levi-Strauss, señala como procedimientos característicos del ritual a la división y la repetición (1988). Así, este autor explica que la acción principal se descompone en una secuencia de multiplicidad de palabras y acciones, y estas microsecuencias, al unirse entre sí sin descanso, es lo que confiere al ritual político una atmósfera especial. Por tanto, se debe señalar que la acción ritual es repetitiva y a menudo redundante. Esta repetición, ya sea de contenidos o de forma, de tiempo o de espacio, le confiere estabilidad al ritual.

3) Tendencia conservadora: La consecuencia directa de este carácter repetitivo es la rigidez y la dificultad que tiene el rito para transformarse. Gran parte de su poder emotivo se debe a esta tendencia conservadora, ya que las representaciones anteriores del ritual reaparecen, en cierta forma, en las reconstrucciones posteriores. Por tanto, se puede afirmar que los cambios en los ritos políticos no se dan de manera progresiva, sino que suelen ser cambios bruscos debidos a unos acontecimientos históricos determinados

4) Dramatización: La transmisión de mensajes a través de la dramatización ritual es mucho más potente que la comunicación verbal. La acción ritual es una actuación muy elaborada y puede utilizar múltiples elementos como: sonidos, música, danzas, colores, gestos, vestidos especiales, etc. Como afirma Gómez García (2002) el ritual "articula gestos, y en ocasiones palabras o cantos, realizados por personas cualificadas, en lugares y tiempos predeterminados y consagrados a tal fin, en los que se utilizan objetos y parafernalias a veces muy sofisticados" (pp. $1-2)$.

Ámbitos. Revista Internacional de Comunicación | ISSN: 1139-1979 | E-ISSN: 1988-5733, №. 50. (2020) 
5) Dimensión temporal: Los rituales abren un umbral de tiempo específico que discurre más aprisa que el ordinario. No deja de ser una fracción de tiempo ordinario, con un principio y un fin, pero donde ocurren muchos acontecimientos significativos. Los rituales fragmentan la vida cotidiana de las personas, dejando a un lado sus quehaceres ordinarios y abriendo un umbral de tiempo especial, más intenso y sacralizado.

6) Orden: otro aspecto fundamental de los rituales políticos es el orden, ya que son eventos organizados. De hecho, y aunque no excluyen momentos o elementos de caos y espontaneidad, el orden en un ritual puede llegar a ser exageradamente preciso. Como defiende Díaz Cruz (1998), los rituales cuentan con reglas y guías prescritas por tradición o convención, que son explícitas y que determinan quién hace qué y cuándo. En cuanto al espacio, los rituales convierten el lugar elegido para su celebración en un sitio especial y sacralizado, donde se escenifican las relaciones de poder entre los presentes, ya que la posición que se ocupa en la acción ritual implica una cierta correspondencia con la que se ocupa en la sociedad. Es este aspecto del ritual el que da sentido al Protocolo, que es el encargado de velar por el orden y el desarrollo de la secuencia del ritual.

\subsection{Aproximación al ritual de toma de posesión}

Como ya se ha demostrado de manera teórica, los rituales políticos no son ajenos a las democracias modernas: los funerales de Estado, las inauguraciones públicas o las fiestas nacionales, son ceremonias que pueden ser analizadas desde esta perspectiva. Si hay un ritual político por excelencia en nuestras sociedades modernas, este es el de la toma de posesión, que se puede definir como el acto por el que una persona se hace cargo de un puesto de responsabilidad en una institución pública. En este sentido, se trata de algo muy extendido en las Administraciones Públicas, que va desde un acto administrativo por el que una persona toma de posesión de su plaza de funcionario, hasta la ceremonia de proclamación del presidente de un territorio. No obstante, las diferencias entre ambos "tipos" de toma de posesión son enormes. Los primeros, son actos legales, burocráticos y privados, que en la mayoría de los casos se limitan a la firma de una serie de documentos oficiales. Los segundos, son actos públicos, con una gran trascendencia mediática, que cuentan con una serie de elementos simbólicos y ceremoniales, están cargados de emoción y hacen referencia a valores trascendentes como la nación, la democracia o el poder. Así, cabe subrayar que este trabajo tiene como objeto de estudio el análisis de las tomas de posesión presidenciales, debido a la relevancia comunicativa que alcanzan este tipo de actos.

Teniendo en cuenta a David Kertzer (1988, la diferencia entre ritual y costumbre es la presencia de símbolos que trascienden lo cotidiano y que dan sentido a una forma de entender el mundo. Así, la toma de posesión de un funcionario es una costumbre, ya que se trata de un acto administrativo que se repite, pero que no está rodeado de símbolos y no evoca valores trascendentes. Por el contrario, la ceremonia de toma de posesión de un presidente, por mucho que se haya repetido a lo largo de la historia, no es un acto más. Se trata de un ritual en sí mismo, ya que en él se manipulan símbolos (banderas, himnos o ejemplares de la Constitución o el Estatuto de autonomía) para investir de autoridad al nuevo líder de una comunidad de personas.

Se trata, en definitiva, de un ejemplo paradigmático de ritual de consenso (Abélès, 1988) en el que, al repetir un mismo ceremonial, con los mismos gestos, las mismas palabras y los mismos símbolos, se pretende escenificar un traspaso pacífico del poder, otorgar legitimidad simbólica al nuevo presidente y transmitirle toda la fuerza histórica de sus antecesores. De este modo,

Ámbitos. Revista Internacional de Comunicación | ISSN: 1139-1979 | E-ISSN: 1988-5733, №. 50. (2020) 
todo el rito de toma de posesión, con sus discursos, sus símbolos y sus actos, está orientado a la emoción y no a la razón.

El objetivo del ritual de la toma de posesión es la construcción de solidaridad, ya que en esta ceremonia se reúnen los principales representantes de la comunidad para reconocer al nuevo gobernante, reforzar los lazos que unen las estructuras sociales y revitalizar a la institución pública. En este punto es interesante tener en cuenta la aportación del profesor Javier del Rey Morató cuando analiza la campaña electoral como un "periodo ritual" que es parte de "la liturgia de la democracia" (1989, p. 172), y mediante el cual se transfiere al orden de la comunicación la agresividad que contiene la lucha por el poder. Siguiendo esta teoría, se puede definir la toma de posesión como la escenificación simbólica, a través de un rito de consenso, de la pacificación social tras el enfrentamiento que supone la campaña electoral. En él, toda la sociedad, incluidos los derrotados, reconocen la victoria y por tanto la autoridad y legitimidad del vencedor. Se trata, por tanto, de un acto vital y trascendente para una sociedad y con el que se pretende desterrar la agresividad con la que se resolvía en tiempos pasados la lucha por el poder.

Sobre este particular, se debe hacer una apostilla -constructiva- a esta teoría, ya que Morató considera que "el calendario de la política implica en su código a toda la sociedad, en la producción de un ritual periódico que culmina -y vuelve a empezar- en el acto de las elecciones" (p. 211). Sin embargo, habría que añadir que, si bien el ritual de las elecciones es parte esencial del proceso democrático, éste termina con la escenificación del traspaso de poderes. Es decir, la liturgia de la democracia termina, y vuelve a empezar, con el ritual de consenso que supone la toma de posesión. Por lo tanto, este último es la culminación, o al menos el epílogo, del ritual de enfrentamiento de las elecciones.

Como se ha podido ver a lo largo de este epígrafe, las tomas de posesión se pueden estudiar desde diferentes perspectivas teóricas. De este modo, existen numerosos trabajos que las han estudiado desde el análisis de los discursos pronunciados por los presidentes (Chirinos y Moleros, 2004; Chumaceiro y Gallucci, 2008; o Frank, 2011; entre otros) o desde la perspectiva que aporta el Protocolo (Pulido, 2003; Mena, 2016; y García, 2018).

\section{OBJETIVOS Y METODOLOGÍA}

Esta investigación parte de la hipótesis de que el acto de toma de posesión de los presidentes autonómicos constituye un auténtico ritual político. Como consecuencia de ello, esta investigación tiene como objetivo principal (OP) realizar un análisis cuantitativo y cualitativo de las ceremonias de tomas de posesión de los presidentes autonómicos españoles. Además de este objetivo primordial también se plantean una serie de objetivos específicos (OE) con los que se pretende orientar el desarrollo de la investigación:

- OE1: Analizar la evolución histórica de las ceremonias de tomas de posesión de los presidentes autonómicos españoles y también la situación actual de este tipo de actos oficiales.

- OE2: Exponer los patrones comunes que aparecen en este ceremonial.

- OE3: Analizar las particularidades y diferencias de estas celebraciones según el ámbito geográfico analizado.

Para aplicar este estudio, la muestra la componen las 203 tomas de posesión de los 106 presidentes autonómicos que han tenido las 17 Comunidades Autónomas, lo que supone el $100 \%$ de los posibles casos. Esto ha sido posible gracias al análisis en profundidad de 92 vídeos 
completos, 41 archivos audiovisuales (audios o pequeñas piezas audiovisuales) y más de 700 ejemplares de prensa escrita. Con este fin, se ha establecido la celebración de las primeras elecciones de cada región como criterio de inicio del marco temporal y el 31 de diciembre de 2019 como cierre. Así pues, la muestra de cada territorio depende del número de convocatorias electorales y de otros factores como las dimisiones o mociones de censura que han ocurrido a lo largo de la historia. Como consecuencia de ello, hay comunidades como Andalucía o la Región de Murcia con 14 casos (6.9\% del total respectivamente) y otras con diez, como Extremadura $(4,9 \%)$.

Con el objetivo de realizar una investigación coherente y sistemática, la metodología empleada para esta investigación es la del análisis de contenido. Se trata de un procedimiento científico muy empleado en las Ciencias Sociales (Silverman, 2016; Krippendorff, 2004; Neuendorf, 2002), que permite reducir grandes cantidades de texto escrito o audiovisual a una serie de variables más limitada con el fin de establecer relaciones entre ellas y desprender significados o interpretaciones (Peña y Lillo, 2012).

Teniendo en cuenta que esta investigación concibe las ceremonias de toma de posesión como un acto de comunicación política, se considera adecuado que se les aplique dicha técnica, registrándolas y codificándolas a través de una serie de variables. Este enfoque es novedoso, puesto que este método no se aplica sobre los documentos periodísticos estudiados, sino sobre la propia ceremonia. Para ello, el diseño de la ficha de análisis corresponde a una estructura de doble enfoque, cuantitativo y cualitativo, y se contemplan 78 variables. El programa estadístico elegido para el procesamiento de los datos obtenidos es el IBM SPSS Statistics, versión 24. La fiabilidad de los intercodificadores se ha calculado mediante la fórmula pi de Scott, alcanzando un nivel de error de 0,98. A continuación, se resumen las principales variables de la ficha de análisis:

Indicadores de Localización:

- Número de ficha

- Año

- Cargo institucional

Análisis general:

- Duración

- Lugar: sede de la Presidencia, sede del parlamento u otros

- Número de asistentes

- Horario: mañana o tarde

- Partido político

- Tipo de acto: pleno del parlamento, austero, solemne o influencia de actos electorales

Fórmula de juramento o promesa:

- Tipo de fórmula: genérica (adapta el Real Decreto 707/1979) o propia

- Tipo de compromiso: juramento o promesa

- Lealtad al rey o la Corona: sí o no 
- Acatamiento de la Constitución: sí o no

- Acatamiento del Estatuto de Autonomía: sí o no

- Mantenimiento del secreto de las deliberaciones: sí o no

Simbología empleada:

- Banderas: de España, de la comunidad autónoma o de la Unión Europea

- Reposteros: de España, de la comunidad autónoma u otros

- Libros ceremoniales: Constitución, Estatuto de Autonomía u otros

- Elementos escenográficos: atril, estrado, mesa presidencial, decoración floral, obras de arte o pantallas de televisión

- Simbología religiosa: Biblia, crucifijo y otros símbolos religiosos

Desarrollo de la ceremonia:

- Llegada de la presidencia: normal o solemne

- Bienvenida: no, voz en off, presentador, presidente del parlamento, representante del Gobierno $\mathrm{u}$ otros

- Lectura del nombramiento: no, voz en off, alto funcionario, presidente del parlamento, secretario de la Mesa u otros

- Juramento o promesa: sí o no

- Himnos: himno nacional o himno autonómico

- Discursos: presidente electo, presidente del parlamento, representante del Gobierno de España, presidente saliente

\section{RESULTADOS}

\subsection{Características generales y evolución histórica}

A partir del análisis comparado de los 203 casos comprendidos en esta investigación, se han podido establecer los rasgos comunes, las particularidades y la evolución histórica de estas ceremonias. Profundizando en las principales características de estas tomas de posesión, hay que señalar que en líneas generales se trata de un acto mayoritariamente solemne (74\%), aunque también se celebra bajo el formato de Pleno del parlamento autonómico (25\%), como ocurre en el País Vasco y la Comunidad Valenciana. Además, hay un reducido número de casos (4\%) que tienen un carácter muy austero y que responden a circunstancias políticas muy concretas, como dimisiones de presidentes, conflictos políticos o crisis económicas. Por otro lado, es destacable que a partir de 2004 se empieza a notar una cierta influencia de los actos electorales en algunas de las tomas de posesión (3\%).

En cuanto a la duración de estos, la media de estas ceremonias es de 25 minutos, pero también se producen considerables oscilaciones entre territorios, dependiendo especialmente del número de discursos que se pronuncien. A este respecto, las más breves son las del País Vasco y la Comunidad Valenciana, al tratarse de plenos con una duración media de 20 minutos al contar con un discurso o ninguno. En el extremo contrario, se sitúa el Principado de Asturias con 
36 minutos de promedio, ya que en ocasiones se producen hasta cuatro intervenciones. Teniendo en cuenta la evolución histórica, hay que resaltar que se observa una tendencia al alza, al pasar, con grandes oscilaciones, de 24 a 30 minutos.

Teniendo en cuenta el horario, se ha podido determinar que existe una gran mayoría de actos que se celebran por la mañana (74\%) y que esta tendencia va en aumento, pasando del $62 \%$ de las primeras ceremonias (1980-1983) hasta el $85 \%$ de las últimas (2016-2019). Esto puede estar relacionado con el objetivo de los gabinetes de comunicación de las presidencias autonómicas de que se cubran estos actos en los informativos del mediodía.

Por otro lado, hay que significar que la mayoría de los casos (46\%) tienen lugar en la sede del parlamento autonómico, especialmente en Galicia, Asturias, Aragón o Comunidad Valenciana, y que existe una tendencia al alza de este tipo de actos, pasando de suponer el $19 \%$ en los primeros años (1980-1983) al 50\% en los últimos (2016-2018). En cuanto a los restantes, el 38\% tienen lugar en la sede de la presidencia regional (Cataluña, Murcia o Islas Baleares) y un $16 \%$ en otros edificios, normalmente con gran valor histórico-artístico, como es el caso del País Vasco.

Terminando con las características generales, es preciso destacar que las tomas de posesión autonómicas son actos con gran afluencia de público, ya que acuden más de 400 personas de media. No obstante, también se han observado grandes oscilaciones entre comunidades, habiendo algunas que superan los 600 participantes (como La Rioja y Cataluña) y otras que no llegan a los 300 (como Canarias, Navarra o País Vasco).

Otro de los aspectos de estas celebraciones que ha sido estudiado es la simbología presente. Tras un análisis pormenorizado de los materiales periodísticos, se ha podido determinar que los elementos más empleados son las banderas, los libros ceremoniales y los reposteros.

- Banderas: esta pieza de tela que sirve como insignia identificativa de cada territorio, es un elemento con una gran potencia simbólica y esto se refleja en que en todas las tomas de posesión de 14 de las 17 comunidades autónomas están presentes tanto la bandera de España, como la de la propia autonomía. En cuanto a las excepciones, hay que destacar que en el País Vasco nunca se utiliza bandera alguna y que en Cataluña se opta por incorporar únicamente la senyera en el $67 \%$ de los casos. Todo esto lleva a que en el $92 \%$ de las tomas de posesión estudiadas se emplee la bandera autonómica y en el $88 \%$ la española. En cuanto a la bandera de la Unión Europea, cabe destacar que tiene una presencia del $24 \%$, aunque también se debe tener en cuenta que España se incorpora a dicho organismo en 1986. De hecho, su presencia aumenta considerablemente con los años, pasando de emplearse en el $5 \%$ de las celebraciones que tienen lugar entre 1988-1991, al 55\% en las de 2016-2019. 


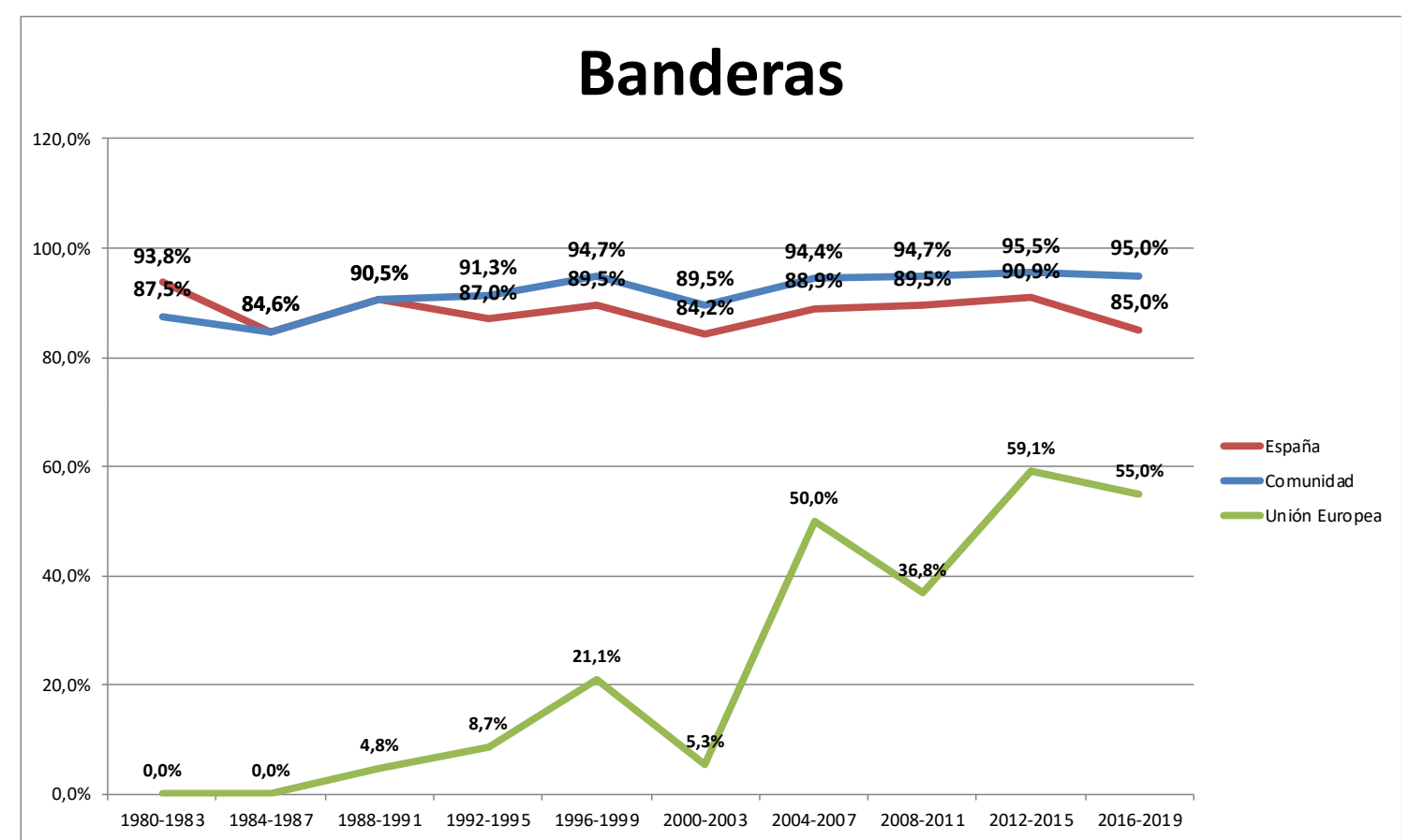

Gráfico 1. Evolución histórica de la presencia de banderas oficiales

Fuente: Elaboración propia.

- Libros ceremoniales: se trata de unos elementos simbólicos de gran importancia en estas ceremonias, ya que es sobre ellos donde el presidente jura o promete su cargo. Así, la Carta Magna está presente en el $87 \%$ de las ocasiones y el Estatuto de Autonomía en el $79 \%$. En cuanto a las particularidades, es resaltable que los presidentes de la Generalitat de Cataluña optan por no utilizar libro alguno y que en el País Vasco sólo se use el Estatuto en el $25 \%$ y que nunca se emplee la Carta Magna. Siguiendo con las diferencias, también es llamativo que en un $9 \%$ de las ceremonias se emplee únicamente el ejemplar de la Constitución, sin la compañía del Estatuto, y esto ocurre especialmente en Castilla-La Mancha y La Rioja.

- Reposteros: el 59\% de estas celebraciones son presididas por un tapiz con el escudo de la comunidad en el centro, algo que ocurre especialmente en Asturias, Comunidad de Madrid y Galicia. Además, hay que señalar que su presencia aumenta con el tiempo, ya que se pasa del 31\% en los primeros años (1980-1983) al 55\% en los últimos (20162019). En cuanto al repostero con el escudo de España, éste acompaña al autonómico tan sólo en el $8 \%$ de las ceremonias estudiadas, principalmente en Extremadura y Comunidad de Madrid.

- Simbología religiosa: estos símbolos, con una importante carga política, están presentes en el $45 \%$ de las ceremonias, en concreto el crucifijo se emplea en el $35 \%$ de los casos, y la Biblia en el $30 \%$. Sobre este particular, hay que resaltar que existe una clara tendencia hacia su desaparición, pasando la Biblia del $38 \%$ en las primeras ceremonias al $5 \%$ en las últimas. En lo que respecta al crucifijo, su evolución es más llamativa, ya que es utilizado en el $44 \%$ de las primeras celebraciones y desaparece por completo en las últimas. Por el contrario, las tomas de posesión en las que no hay elementos confesionales evolucionan con el paso del tiempo del $50 \%$ al $85 \%$. 


\section{Simbología religiosa}

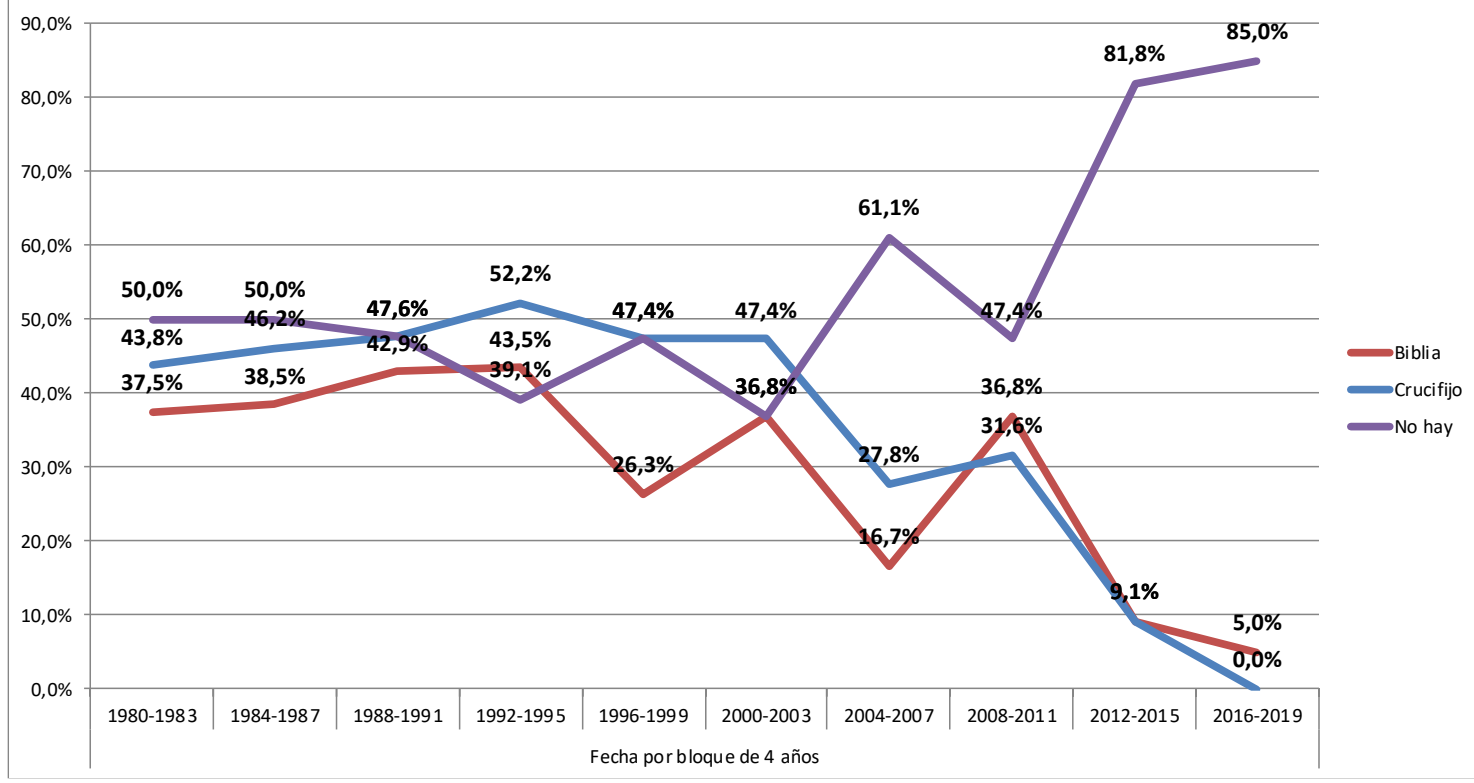

Gráfico 2. Evolución histórica de la presencia de simbología religiosa

Fuente: Elaboración propia.

- Elementos escenográficos: entre el mobiliario empleado para completar la puesta en escena de estos actos, hay que destacar la mesa de juramento o promesa sobre la que se colocan los libros ceremoniales y la simbología religiosa (85\%); el estrado empleado para elevar la zona de honor (77\%); y un atril para los discursos (73\%). Otros ornamentos que se usan en algunas celebraciones son la decoración floral (51\%), la mesa presidencial (38\%) u obras de arte (6\%). Si se tiene en cuenta la evolución histórica, se han detectado tendencias considerables, ya que tanto el atril como el estrado aumentan muy considerablemente su presencia, pasando respectivamente del $25 \%$ y $50 \%$ en los primeros años (1980-1983) al 90\% en los últimos (2016-2019). El caso contrario se produce en relación con la mesa presidencial, que pasa del $62,5 \%$ en el primer periodo al $15 \%$ en el último. Igualmente, hay que significar que en los últimos años se han incorporado elementos más propios de los actos electorales como pantallas de televisión (3\%).

A lo largo de esta investigación, también se han analizado las autoridades que están presentes en la presidencia de las tomas de posesión autonómicas y para ello se ha realizado un análisis pormenorizado del material audiovisual localizado. De este modo, se debe apuntar que son tres las personas que con mayor frecuencia están presentes:

- Presidente electo: se trata del protagonista de la ceremonia, ya que es quien toma posesión del cargo, y está presente en el $88 \%$ de las mismas. Sólo se omite en aquellos casos en los que queda diluida la presidencia o en los que ésta se celebra bajo el formato de Pleno del Parlamento, algo que ocurre especialmente en la Comunidad Valenciana.

- Presidente del Parlamento: aunque se encuentra en el $81 \%$ de los casos, su presencia oscila mucho entre comunidades, ya que siempre se incorpora a la cabecera del acto en 
siete de ellas (País Vasco, Cataluña, Galicia, Asturias, La Rioja, Comunidad Valenciana y Aragón), pero apenas lo hace en otras como Islas Baleares o Andalucía.

- Representante del Gobierno de España: su presencia en la presidencia de estos actos es del $66 \%$ y depende mucho de la situación política de cada territorio y del formato de la ceremonia. En cuanto a esto último, hay que destacar que nunca está presente en las tomas de posesión vascas y valencianas, ya que estos actos se celebran bajo el formato de pleno del parlamento. En cuanto a la tendencia histórica, hay que resaltar que pasa de participar de la cabecera en el $88 \%$ de los primeros años al $60 \%$ de los últimos.

En otro orden de cosas, este trabajo de investigación también categoriza sistemáticamente los elementos, gestos e intervenciones que componen el esquema ceremonial de estos actos institucionales:

La mayoría de los casos estudiados (84\%) comienzan con una llegada ordinaria de las autoridades que conforman la presidencia del acto. En cuanto al 16\% restante, la celebración empieza con una llegada solemne de estas personalidades y esto ocurre principalmente en el País Vasco, Cataluña, Navarra y Aragón. A continuación, en el 77\% de las ceremonias se ofrecen unas palabras de bienvenida, un gesto que se va acentuando con los años, ya que se pasa del $50 \%$ en $1980-1983$ al $100 \%$ a partir de $2008-2011$. Sin embargo, hay que puntualizar que la persona que pronuncia la bienvenida varía considerablemente dependiendo del territorio. A este respecto, mayoritariamente es el presidente del Parlamento quien lo hace (41\%), seguido de una voz en off (22\%). El siguiente elemento es la lectura del real decreto de nombramiento, que se da en la práctica totalidad de las ceremonias estudiadas (94\%). En cuanto a las excepciones, hay que señalar que diez de estos doce casos tienen lugar en la Comunidad Valenciana y en ellos, aunque no se lee, sí se menciona este texto legal. En cuanto a la persona que se encarga de ello, hay que señalar que esto varía claramente dependiendo del territorio.

A continuación, se produce el juramento o promesa, el elemento central de estas ceremonias, que tiene lugar en el $100 \%$ de los casos estudiados y que consiste en la pronunciación por parte del presidente de una fórmula preestablecida mediante la cual asume el cargo y se compromete, en la mayoría de los casos, a acatar la legalidad establecida. En este sentido, hay que resaltar que en el $71 \%$ de los casos se trata de una adaptación del texto establecido por el Real Decreto 707/1979. En el 29\% restante se emplean fórmulas propias de la comunidad correspondiente, algo que ocurre especialmente en País Vasco, Galicia, Asturias, Comunidad Valenciana y Navarra.

En cuanto a los elementos que componen la fórmula ceremonial hay que resaltar que los presidentes autonómicos manifiestan su lealtad al rey o a la Corona en el $79 \%$ de los casos. En cuanto al $21 \%$ que no lo hace, esto ocurre principalmente en Galicia, Asturias y Navarra. Profundizando en la mención al monarca, existe una tendencia a la baja al pasar del $94 \%$ de los primeros años (1980-1983) al 65\% del último periodo (2016-2019). Además, se debe reseñar que el otro elemento central de estos textos es el acatamiento de la Constitución y el Estatuto de Autonomía, algo que ocurre en el $98 \%$ de los casos estudiados. De hecho, las únicas excepciones son las dos primeras ceremonias del País Vasco (1980 y 1984) y las dos últimas de Cataluña (2016 y 2018).

Para terminar con las características de esta fórmula, hay que destacar que el $53 \%$ de los presidentes optan por la promesa y el $47 \%$ por el juramento. Sin embargo, hay diferencias considerables entre comunidades, ya que en algunas se prefiere mayoritariamente prometer (Cataluña, Asturias o Extremadura) y otras jurar (País Vasco, Cantabria o Castilla-León). A este 
respecto, no existe un patrón claro en lo relativo a su evolución histórica, más allá de que en los ocho primeros años (1980-1987) hay una clara mayoría de presidentes que optan por prometer el cargo y que a partir de ahí las diferencias se reducen.

Por otra parte, los discursos suponen el otro gran apartado de estas celebraciones, al tener lugar en el $92 \%$ de las tomas de posesión estudiadas, y son quienes aportan la mayor carga política a las mismas. De hecho, diez de las 16 ocasiones en las que no se producen intervenciones corresponden al País Vasco, cuyo ceremonial tradicionalmente los omite, y las otras seis responden a situaciones políticas muy determinadas. Ahondando en las autoridades que pronuncian los discursos, la intervención que se produce con mayor frecuencia (92\%) es la del presidente electo, seguida de la del representante del Gobierno de España (55\%) y la del presidente de la Cámara autonómica (45\%). De estas tres, en la que más oscilaciones se producen es en la del enviado estatal, ya que hay algunos territorios en los que éste nunca interviene (País Vasco, Cataluña o Comunidad Valenciana) y otros en los que lo hace con gran frecuencia (Asturias, La Rioja, Cantabria y Navarra). Además, en términos globales se observa que su participación sufre una tendencia a la baja, pasando del $81 \%$ en $1980-1983$ al 55\% en 2016-2019.

En cuanto al resto de componentes del ceremonial de las tomas de posesión hay que destacar que en el $40 \%$ de ellas se interpreta el himno autonómico y en el $13 \%$ el de España. Además, en ciertos casos se incorporan piezas musicales (11\%) o danzas tradicionales (5\%). Otro gesto por resaltar es que en el $4 \%$ de los casos estudiados se observa que el resto del Gobierno autonómico toma posesión junto al presidente. En estas nueve ceremonias, que se concentran entre 1980 y 1987, se podría decir que la figura del presidente se reduce a la de un primus inter pares.

\subsection{Influencia de los partidos políticos}

Además de todo ello, también se ha estudiado si la pertenencia del presidente electo a un partido u otro influye en el desarrollo de la celebración. Así, las fuerzas políticas que se tienen en cuenta son PSOE, PP, PNV y CiU, puesto que el resto no representan una muestra lo suficientemente amplia como para aportar datos significativos. En cuanto a las características generales de las ceremonias estudiadas, es importante destacar que los partidos de ámbito estatal tienen mucha más variedad que los partidos nacionalistas, ya que estos sólo están presentes en un territorio y tienen menos casos a estudio.

En la categoría en la que sí se han encontrado resultados destacables es el tipo de compromiso elegido por los presidentes autonómicos para asumir su cargo. En cuanto a los partidos de ámbito nacional, en el PSOE hay una clara mayoría de dirigentes que optan por prometer (84\%), mientras que en el PP ocurre lo contrario siendo mayoritarios quienes optan por jurar (79\%). Por lo que respecta a los partidos nacionalistas, las diferencias son más llamativas, ya que todos los lehendakaris del PNV juran su cargo, frente a todos los presidents de CiU que lo prometen. Otro dato reseñable es que las cifras de las cuatro fuerzas son bastante parejas en lo relativo al compromiso con el rey o la Corona, ya que lo hacen el $82 \%$ de los presidentes del PP y del PNV, el $80 \%$ de los de CiU y el $79 \%$ del PSOE. 


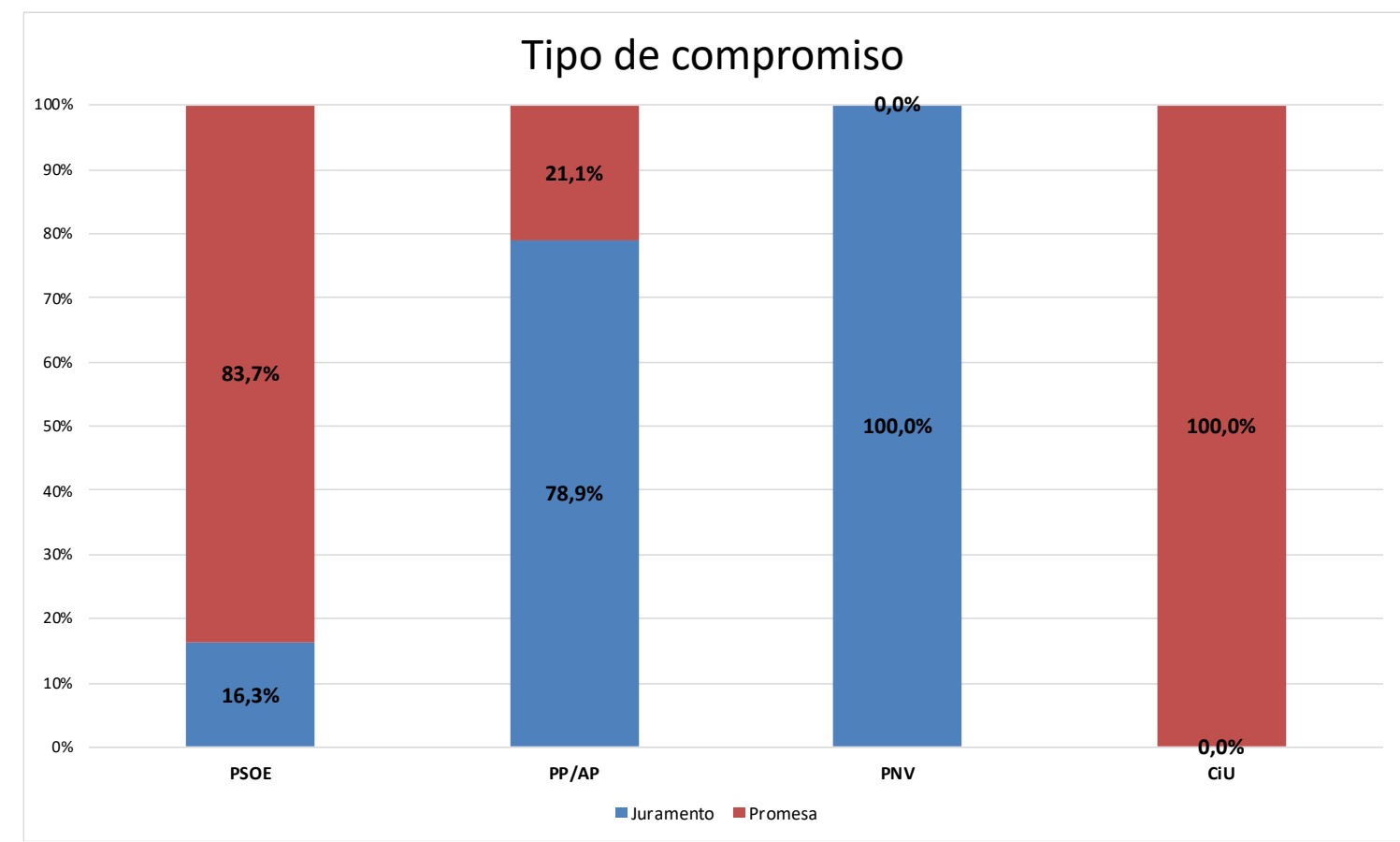

Gráfico 3. Tipo de compromiso por partido político

Fuente: Elaboración propia.

Si se tiene en cuenta la simbología presente, las diferencias entre los partidos estatales y los partidos nacionalistas también son claras. Así pues, mientras que en la práctica totalidad de las ceremonias del PSOE y PP están presentes las banderas de España (97\% y 99\%) y de la comunidad autónoma ( $98 \%$ y $99 \%$ ), esto no ocurre en el caso de los partidos nacionalistas. No obstante, el comportamiento de éstos es diferente, puesto que el PNV opta por no incorporar bandera alguna, mientras que CiU usa la bandera catalana en el $50 \%$ de los casos y la enseña nacional solamente en 1980, lo que supone el 10\%. En cuanto a la bandera de la Unión Europea, las cifras son más divergentes al ser empleada en el $30 \%$ de las tomas de posesión socialistas, el $18 \%$ de las populares y en ningún caso por parte de los dos partidos nacionalistas. Otro de los símbolos en los que se nota la divergencia entre partidos de ámbito estatal y los nacionalistas es el uso de ejemplares de la Constitución y el Estatuto de Autonomía. En cuanto al PP y PSOE, se emplean en proporciones similares ambos libros ( $96 \%$ el primero y $84 \%$ el segundo). Frente a ello, en las ceremonias de CiU no se emplea ninguno y en las del PNV tan sólo se incorpora el Estatuto a partir de 2009 , lo que supone el $18 \%$.

Por otro lado, también se observa una influencia de los partidos políticos en cuanto al uso de simbología religiosa. Así, el PNV es la fuerza que más veces incorpora este tipo de símbolos, ya que tanto la Biblia como el crucifijo están presentes en el $64 \%$ de sus ceremonias; seguida del $\mathrm{PP}$, que lo hace en el $46 \%$ y el $51 \%$ respectivamente. En el extremo contrario está CIU, que no las utiliza; y PSOE, que sólo emplea la Biblia en el $9 \%$ de los casos estudiados y el crucifijo en el $13 \%$. 


\section{Simbología religiosa}

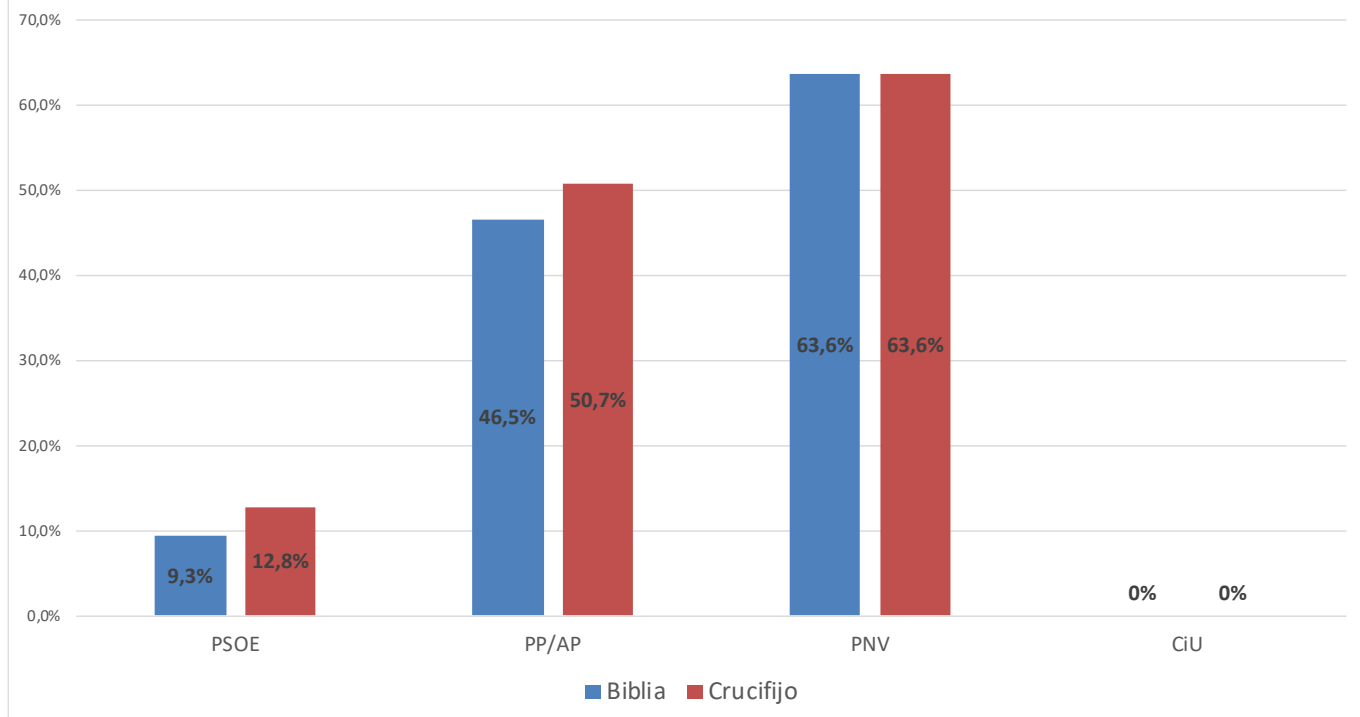

Gráfica 4. Presencia de simbología religiosa por partido político Fuente: Elaboración propia.

Ahondando en las autoridades presentes en la cabecera del acto, hay que significar que el presidente electo y el presidente del parlamento están presentes en todos los casos de PNV y CiU, mientras que la cifra baja en las ceremonias del PP ( $87 \%$ y $89 \%$ respectivamente) y del PSOE ( $88 \%$ y $71 \%$ ). Sin embargo, donde hay más divergencias es en la presencia del representante del Gobierno de España, ya que es en las ceremonias del PSOE donde más se le incorpora (78\%), frente a CiU (70\%), PP (68\%) y PNV (0\%). De este modo, es reseñable que el Partido Popular, siendo un partido de ámbito estatal, tenga un promedio más bajo que CiU. Además, estas diferencias se agravan si se tiene en cuenta si éste interviene en el acto, puesto que en las celebraciones de PNV y CiU jamás interviene el enviado del Ejecutivo de la Nación. Además, entre los partidos de ámbito nacional también existen divergencias considerables, ya que éste participa en el $72 \%$ de las tomas de posesión de presidentes socialistas, frente al $52 \%$ de las de los populares. 


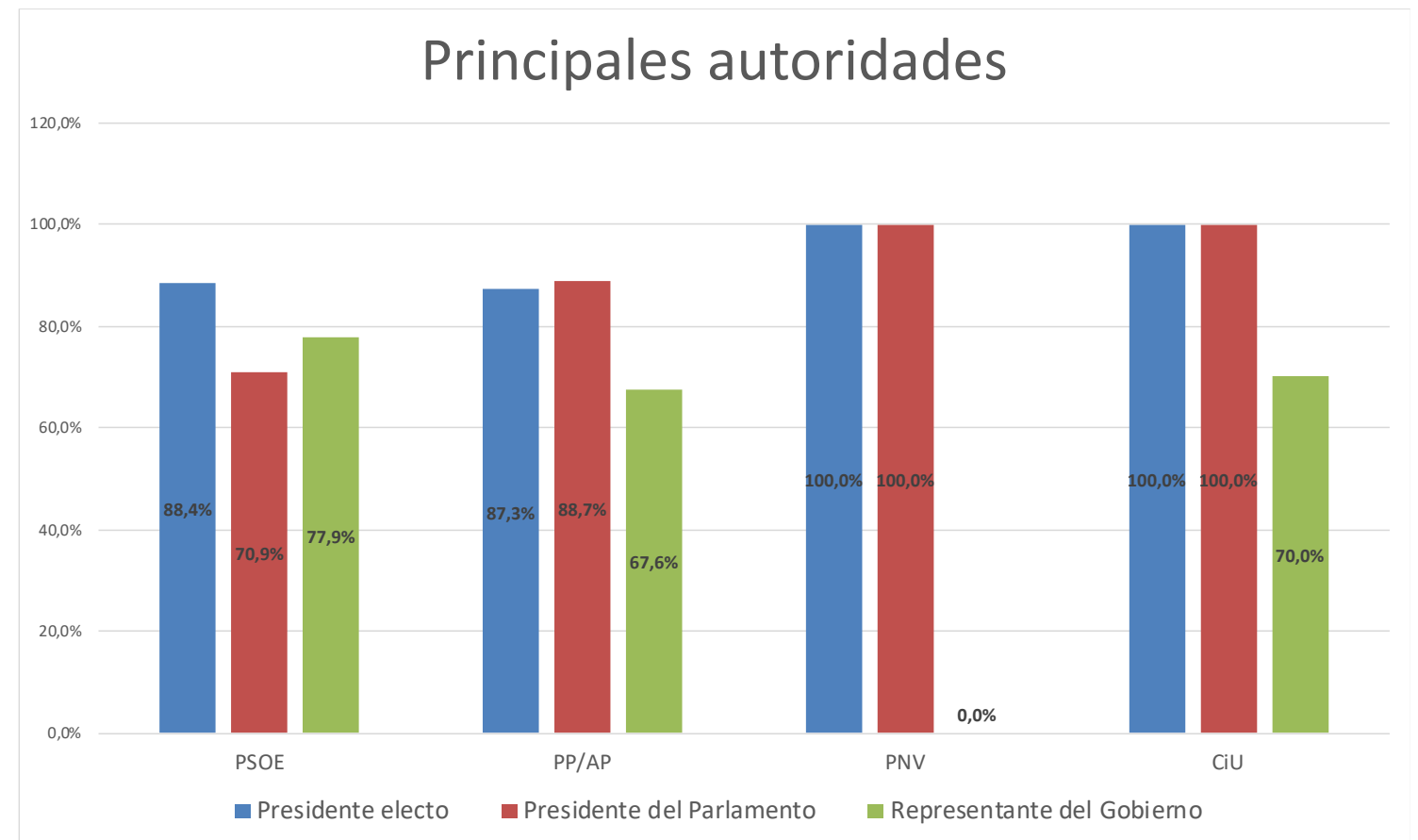

Gráfico 5. Presencia de autoridades por partido político

Fuente: Elaboración propia.

\section{CONCLUSIONES}

Teniendo en cuenta la revisión teórica y los resultados obtenidos, esta investigación refuerza alguna de las conclusiones de trabajos precedentes, que apuntan a la importancia del ceremonial y el simbolismo en los actos de toma de posesión (Pulido, 2003; Mena, 2016; y García, 2018). Sin embargo, una de las novedades que este trabajo es que ahorma un entramado teórico en torno al concepto de ritual político y para ello se acude a autores internacionales como Abélès (1988), Kertzer (1989) y Balandier (1994), que defienden una concepción amplia del fenómeno del ritual, desligándolo de la religión y relacionándolo con el mundo de la política moderna.

Atendiendo a los resultados obtenidos tras el análisis de contenido de los 203 casos a estudio, podemos afirmar que la hipótesis de investigación queda ratificada y que la toma de posesión presidencial es un ejemplo paradigmático de ritual político que persiste en las democracias actuales. En este sentido cabe destacar:

- En la práctica totalidad de las tomas de posesión presidenciales se utilizan los símbolos oficiales que representan al territorio, como son las banderas, los escudos o los himnos. Mediante esta manipulación simbólica, se busca vincular al presidente que asume el poder con la institución que liderará. Este mecanismo es el que sirve para reforzar la legitimidad del gobernante frente a la sociedad.

- El acto de toma de posesión presidencial es una ceremonia que se basa en la estandarización y en la repetición a lo largo del tiempo de unos gestos, palabras y formas determinadas. La carga emotiva que aporta la tradición sirve para representar la 
continuidad histórica de la institución, más allá de la temporalidad de quienes la presiden.

- Esta ceremonia está dramatizada y para ello se utilizan piezas musicales, se aporta una decoración extraordinaria o se emplean palabras que aumentan la solemnidad a la misma. Se trata de un evento extraordinario, que rompe con la actividad cotidiana de los participantes y que abre por tanto un umbral de tiempo especial y más intenso.

- Se trata, en definitiva, de un acto que escenifica la pacificación de la sociedad a través de la legitimación del poder del nuevo gobernante. Esto ocurre tras la campaña electoral, un periodo de confrontación entre partidos políticos que divide a la sociedad en auténticos bandos enfrentados.

En cuanto a los objetivos planteados, al analizar el OE1 se observa que existen determinadas tendencias históricas en cuanto al ceremonial, la simbología y las autoridades presentes en las tomas de posesión presidenciales. Si bien hay elementos en los que influye más la particularidad de cada territorio, se han podido establecer patrones como el aumento de la duración del acto y el número de asistentes o la tendencia a realizar estos actos por la mañana y en sede parlamentaria. En otro orden de cosas, también hay que apuntar a que se produce un aumento constante de la riqueza simbólica de estas celebraciones, al incorporar a las habituales banderas de España y de la comunidad, otros elementos como el repostero con el escudo autonómico o la bandera de la Unión Europea. Frente a ello, hay una clara tendencia a la eliminación de los símbolos religiosos como el crucifijo o la Biblia. En cuanto a la escenografía, se tiende a incorporar elementos que mejoran la comunicación del evento como son el atril o el estrado y se eliminan otros como la mesa presidencial. Por último, hay que reseñar que, en lo que respecta al ceremonial, se observa la incorporación de gestos como la bienvenida a los asistentes o los himnos autonómicos, el aumento de la importancia del presidente autonómico frente al representante del Gobierno de España.

En cuanto al segundo objetivo específico, los datos indican que en todas las tomas de posesión analizadas hay un elemento ceremonial común: el acto de juramento o promesa del presidente. Se trata del elemento nuclear de este acto que, a pesar de que se haga de una manera más o menos solemne, se repite en todos los casos estudiados. Este gesto consiste en la formulación por parte del presidente de un texto previamente determinado por el que asume las obligaciones de su cargo. A partir de este punto, en la gran mayoría de las ocasiones se añaden otros gestos ceremoniales como la lectura del decreto de nombramiento o los discursos. Otra de las características comunes es la utilización de símbolos oficiales, tales como banderas, escudos, himnos; o libros ceremoniales, como la Constitución, el Estatuto de Autonomía o la Biblia. Dependiendo de cada territorio, de la situación política o del momento histórico, se emplean en mayor medida unos u otros. Además, también está generalizada la presencia de autoridades, bien en la presidencia del acto o bien como invitados al mismo, que con su asistencia respaldan al nuevo gobernante y legitiman su poder.

Profundizando en el último objetivo, se corrobora que existen considerables diferencias entre cada uno de ellos. De hecho, hay tomas de posesión muy rituales, como las del País Vasco, frente a otras más austeras, como las de la Comunidad de Madrid. También hay algunas que son muy estables, como las del Principado de Asturias, frente a otras con una constante inestabilidad, como ocurre en Canarias. Además, hay tomas de posesión de carácter parlamentario, como las de Galicia o Aragón; otras de tipo ejecutivo, como las de Cataluña; y algunas que se celebran bajo el formato de Pleno del Parlamento, como ocurre en la Comunidad Valenciana. En 
definitiva, existe una gran riqueza ritual y pluralidad, ya que se adapta el ceremonial y la simbología de la toma de posesión a las particularidades histórico-políticas de cada territorio.

Esta investigación aporta nuevas evidencias de la importancia del uso de símbolos, gestos ceremoniales y rituales dentro de la comunicación política moderna. Además, la implementación de la metodología del análisis de contenido a la propia celebración, entendida como acto comunicativo, permite identificar nuevas oportunidades investigadoras que invitan a seguir estudiando los actos institucionales desde esta nueva perspectiva.

En este sentido, sería interesante comparar, con el objetivo de determinar similitudes y diferencias, las tomas de posesión autonómicas con la del presidente del Gobierno y con la proclamación de los reyes Juan Carlos I y Felipe VI. También aportaría información valiosa la aplicación de la metodología de este estudio a diferentes ceremonias políticas de carácter solemne, como las aperturas legislativas, la celebración de los días oficiales de cada territorio o los funerales de Estado.

\section{Referencias}

Abélès, M. (1988). Modern Political Ritual: Ethnography of an Inauguration and a Pilgrimage by President Mitterrand. Current Anthropology, 29(3), pp. 391-404. Recuperado de: https://www.jstor.org/stable/2743455

Balandier, G. (1994). El poder en escenas. Barcelona: Paidós.

Chirinos, A. \& Molero, L. (2007). La imagen del yo y del otro: construcción de identidades en los discursos de toma de posesión de los presidentes de Venezuela y Brasil. Boletín de Lingüística, no XIX(27), pp. 70-93. Recuperado de: http://ve.scielo.org/scielo.php?script=sci_arttext\&pid=S079897092007000100003

Chumaceiro, I. \& Galluci, M. (2008). La noción de democracia en los discursos de toma de posesión de Hugo Chávez y Álvaro Uribe. Revista Internacional De Lingüística Iberoamericana, no 6(2 (12)), pp. 21-31. Recuperado de: https://www.jstor.org/stable/41678349

Del Rey Morató, J. (1989). La comunicación política. El mito de las izquierdas y las derechas. Madrid: EUDEMA.

Díaz Cruz, R. (1998). Archipiélago de rituales: Teorías antropológicas del ritual. Barcelona: Anthropos.

Durkheim, E. (1982). Las formas elementales de la vida religiosa. Madrid: Ediciones Akal.

Frank, D.A. (2011). Obama's Rhetorical Signature: Cosmopolitan Civil Religion in the Presidential Inaugural Address, January 20, 2009. Rhetoric \& Public Affair, no 14(4), pp. 605-630. Recuperado de: https://www.jstor.org/stable/41935240

Fuente, C. (2005). El Protocolo Oficial. Las instituciones españolas del Estado y su ceremonial. Madrid: Ediciones Protocolo.

García Rodríguez, V. (2018). Renovación protocolaria: papel de los símbolos religiosos en actos de toma de posesión. Journal of the Sociology \& Theory of Religion, 7, pp. 147-171. Recuperado de: https://doi.org/10.24197/jstr.0.2017.147-171

Gómez García, P. (2002). El ritual como forma de adoctrinamiento. Gazeta de Antropología, 18. Recuperado de: http://hdl.handle.net/10481/7395

Kertzer, D. (1988). Rituals, Politics and Power. New Haven: Yale University Press.

Krippendorff, K. (2004). Content Analysis: An Introduction to Its Methodology. Thousand Oaks: Sage Publications.

Laforet, J. (2013). El protocolo judicial en España (Tesis doctoral). Universidad de Las Palmas de Gran Canaria. Recuperado de: http://hdl.handle.net/10553/16276

Leach, E. R. (1977). Sistemas políticos de la alta Birmania: Estudio sobre la estructura social Kachin. Barcelona: Anagrama. 
Marín Calahorro, F. (2000). Protocolo y comunicación: Los medios en los actos públicos. Barcelona: Ediciones Bayer Hermanos.

Mena, T. (2016). Toma de posesión de los presidentes del Gobierno de Navarra en sede parlamentaria. Revista de Estudios Institucionales, no 3(4), pp. 78-89. Recuperado de: https://doi.org/10.5944/eeii.vol.3.n.4.2016.18380

Neuendorf, K. A. (2002). The Content Analysis Guidebook. Thousand Oak: Sage.

Otero Alvarado, M. T. (2011). Protocolo y empresa: El ceremonial corporativo. Barcelona: UOC.

Peña y Lillo, M. (2012). La importancia del acuerdo entre codificadores para el análisis del contenido. Comunicación y Medios, no 25, pp. 47-56. Recuperado de: https://comunicacionymedios.uchile.cl/index.php/RCM/article/view/24521

Pulido Polo, M. (2003). Imagen y comunicación en los actos de toma de posesión. Laurea Hispalis: Revista internacional de investigación en relaciones públicas, ceremonial y protocolo, 2 . Recuperado de: http://hdl.handle.net/11441/52736

Sierra Sánchez, J. (2008). Protocolo: herramienta comunicativa persuasiva y simbólica. Zer - Revista de Estudios de Comunicación, 13(24), 337-361. Recuperado de: http://www.ehu.es/zer/hemeroteca/pdfs/zer24-16-sierra.pdf

Silverman, D. (2016). Qualitative research. Londres: Sage.

Thompson, J. B. (2003). La transformación de la visibilidad. Estudios públicos, 90, pp. 273-296. Recuperado de: https://www.cepchile.cl/cep/estudios-publicos/n-61-a-la-90/estudios-publicos-n-90/latransformacion-de-la-visibilidad 